\title{
Models and devices for a multimodal study of the human phonatory apparatus: Technological results and clinical applications
}

Speech is the primary means of communication between humans and results from complex interaction between the vibration of the vocal folds at the larynx and the movements of the voluntary articulators (mouth tongue, jaw, etc.).

A primary purpose of voice analysis is to extract features or parameters that represent relevant characteristics of the acoustic waveform.

First studies go back to the 18th century but strong interest was shown in speech characteristics in the early 20th century along with the growth of the telecommunications industry. Efforts were made to develop tools for speaker identification and for background noise removal. Voice quality assessment was firstly developed for speech coding or synthesis and for speech enhancement. The need for such testing methods became apparent in the 1950s along with the development of new analogue communication systems.

More recently research has focussed on biomedical applications. The aim of voice analysis in the biomedical field being quite different from that of telecommunications, different approaches were developed. Specifically, the acoustical analysis and modelling of the voice source and the vocal tract in healthy and pathological voices are among the main fields of research. The aim is that of extracting the voice characteristics (fundamental frequency and vocal tract resonance frequencies) together with their deviation from "healthy conditions'. Degradation of voice, generally called hoarseness, is in fact one of the major symptoms of benign laryngeal diseases such as polyps or nodules but is often the first symptom of neoplastic diseases such as laryngeal cancer.

The acoustical properties of pathological voice are commonly investigated in terms of perturbations in the fundamental frequency (jitter) and amplitude (shimmer) as well as looking for spectral irregularities through measures of harmonics-tonoise-ratio (HNR). Though all such parameters occur to some extent in normal voices, beyond specified thresholds they can be identifiers of vocal pathology. As for the acoustical analysis, the need for quantifiable measures of voice as related to treatments and medical or surgical intervention has led to a search for acoustic correlates of dysphonic severity. An objective measure of the 'noise level', intended as a measure of voice quality, is of great relevance in biomedical applications. In their daily practice, laryngologists require an objective measure of hoarseness and a scale to compare results of various treatments. Perceptual scales are commonly used, but their cor- relation to objective indexes is still an open problem. Reliable models of the human voice can contribute to solve this problem.

Models are inherently a simplified version of the real physical mechanism, derived from physical principles (mechanical, electrical) and acoustical properties (e.g. multitube resonances) of voice and speech, which are in turn based on anatomy and physiology of the vocal apparatus. Recently, sophisticated versions of biomechanical models have been developed to describe the shape and dynamics of the vocal apparatus more accurately. Thanks to the increasing computing capabilities, the resulting mathematics became tractable and, if the boundary conditions are properly chosen, reliable parameters of the model can be obtained.

Voice analysis is mainly performed in the time, frequency, wavelet and cepstral domains. Classical methodologies have been and are applied for voice analysis but new ones were developed and compared. The so-called non-parametric frequency domain analysis is the most widely used, as it allows fast computations by means of FFT-based algorithms. However, its applicability and resolution capability are linked to the length of the data window under analysis: the shorter the time window the lower the frequency resolution. This is particularly critical for irregular voice signals that are stationary on very short data frames of some tens of milliseconds or high-pitched ones such as e.g. newborn infant cry and the singing voice. Time domain analysis aims at describing the signal dynamics by means of appropriate linear or non-linear models. Due to its high-resolution characteristics, the linear approach that makes use of AR models (linear prediction (LP)) overcomes problems encountered with classical frequency domain analysis giving reliable models of the speech waveform dynamics, though generally at the expense of more cumbersome algorithms. Also, both continuous and discrete wavelet transforms (CWT and DWT respectively) can be successfully applied to the estimation of F0 and formants. The advantage of the waveletbased methods is their capability of performing a multiresolution analysis with a very low computational burden, thus they are particularly suited for quasi-stationary voice signals. The drawback is the semi-empirical choice of the mother wavelet. Cepstral analysis allows the excitation sequence at the glottis to be separated from the vocal tract impulse response, which are convolved in time. Thus, fundamental frequency and formants can be recovered from the transformed signal in the quefrency domain. The 
method is fast and simple but suffers from the same drawbacks of the frequency domain approach as far as resolution is concerned. Moreover, the choice of the lifter is critical. Finally, the application of other non-linear analysis techniques to vocal emissions place more realistic assumptions on the voice production mechanism being based on non-linear dynamical systems theory at the expense of more complex algorithms. Advanced analysis techniques include chaotic models, hidden Markov models, neural networks, and other sophisticated tools.

In addition to studying the audio signal recorded with a microphone, it is worth mentioning the somewhat indirect measure given by the electroglottograph (EGG), a device that provides the noninvasive measurement of the degree of contact between the vibrating vocal folds during voice production. Finally, new numerical methods and devices for image analysis can give more information than usual stroboscopy, that provides the standard view of the larynx. In fact, due to its limited frame rate stroboscopy cannot provide enough details to evaluate highly irregular vocal fold vibrations. Videokymography (VKG) registers the movements of the vocal folds with a high time resolution on a line perpendicular to the glottis, thus it was shown very useful in severely dysphonic patients with strong aperiodic vocal folds vibration. An increased use of other high-speed device in clinics and research laboratories, as well as improved technical capabilities in computer software and hardware and imaging techniques, has allowed detailed views of the vocal folds in motion. Quantitative evaluation of asymmetries and dysperiodicities is made available through increasingly refined software tools.

Along with traditional statistical analysis, new and powerful classification techniques are increasingly applied to differentiate voice signals coming from subjects of different gender, age, native language and different pathologies, to automatically assess the voice quality of patients after surgery or treatment.

The extensive research in modelling and analysis of the human voice gave rise to a huge number of indexes, both perceptual and objective, as well as different measures of the same quantity (such as e.g. jitter and noise). This still prevents a unified comparison of results and there is a need for a standardisation of acoustic measures. Moreover, standard data bases for voice analysis and pathology classification are still missing. Recently reliable synthetic signals are being developed to test software tools.

In addition to adult's voice analysis, it is worth noting that all the above mentioned approaches are increasingly applied in relatively new research areas such as non-speech vocal emissions like newborn infant cry, coughing and snoring, related to physiological and linguistic development, obstructive apnoea and asthma, respectively.

From this short introduction, it emerges that the field of speech and voice analysis is an inherently multidisciplinary one. Signals and images come both from newborns and adults and for a large number of pathologies or diseases useful information can be gained from accurate models and analysis. Hence, co-operation between biomedical engineers, clinicians, physicians, mathematicians and psychologists, is not only desirable but necessary as research not only focus on speech production, hearing and linguistic structure but explores and probes the complex interrelations between these areas.

The MAVEBA biannual series of Workshops held in Firenze, Italy, and never discontinued over the years, focuses on all these themes. It came into being in 1999 from the need, particularly felt by the organizers to share know-how, aims and results between areas that until then seemed quite distinct such as bioengineering and medicine. Therefore, its first aim was to stimulate contacts between specialists from different fields active in research and industrial developments in the area of voice signal analysis for biomedical applications. The scope of the Workshop includes all aspects of voice modelling and analysis, ranging from basic research to all kinds of biomedical applications, devices and related established and advanced technologies.

Over the years MAVEBA has reached full maturity and the initial issues of the workshop have grown and spread also in other aspects of research such as occupational voice disorders, singing voice, neurology, rehabilitation, image and video analysis with applications ranging from the newborn to adult, elderly and singers. In fact, there has been a continuous parallel expansion both in clinical research and in technology devoted to this field leading to an increasing interaction between researchers in technological and clinical disciplines, aiming at providing a common basis of knowledge for future research in this exciting area of biomedical investigation.

Not only multidisciplinary but also multimodality is a major keyword of MAVEBA: in fact different methodologies that relate both to the analysis of signals and of images for the study of the human vocal system are increasingly developed and successfully compared. Combining methods and skills is thus the key to the best results.

Under these perspectives, over the years well-known specialists from all over the World and dealing with any discipline related to voice come to Firenze and attend the MAVEBA Workshop giving their contribution with free papers, invited lectures and special session, offering participants a deeper insight into relevant aspects and results. Young researchers have the opportunity to discuss with specialists and their best paper are awarded and some granted by the BSPC Journal.

As for the past editions, this SI collects peer reviewed contributions that are extended versions of papers presented at the MAVEBA 2015 Workshop whose proceedings are available since 2003 both in printed and open access format (with ISBN code) at http://www. fupress.com/ricerca?q=maveba.

Exceptionally, in 2015 the MAVEBA workshop was held together with the Pan European Voice Conference (PEVOC) and the Collegium Medicorum Theatri meeting (CoMeT), whose abstract book is printed by FUP: http://www.fupress.com/ricerca?q=pevoc.

Specifically, this Special Issue collects ten papers that deal with the main aspects of biomedical applications of voice analysis, both methodological and applicative. An overview is presented here.

The first two papers mainly concern methodological and modelling aspects, tested both with synthesized voices and using human voice signals.

The first paper from Alzamendi et al. "Modeling and joint estimation of glottal source and vocal tract filter by state-space methods" investigates state-space methods to enhance the joint estimation of the glottal source and the vocal tract information. First, a state-space voice model is formulated considering the stochastic glottal source ruled by a stochastic difference equation that allows to accurately capture perturbations occurring at glottal level. Then, combining this voice model and the state-space framework, an inverse filtering method is developed that allows to jointly estimate both glottal source and vocal tract filter. The performance of this method is tested both with synthesized voices and using human voice signals. The results demonstrate that accurate estimates of the glottal source and the vocal tract filter can be obtained over several scenarios. Moreover, the method is shown to be robust with respect to different phonation types.

The paper from Silvia Orlandi et al. "Testing software tools for newborn cry analysis using synthetic signals" concerns the challenging issue of fundamental frequency (F0) and formant frequencies estimation in the high-pitched newborn cry. The acoustical analysis of the infant cry has the advantage of being a cheap 
and contactless approach that might assist the clinical specialist in the detection of abnormalities in infants with possible neurological disorders. The paper compares and tests three methods, one freely available online and the other two specifically built using two different approaches: autoregressive models and wavelets. The comparison is performed on synthetic signals coming from a synthesized developed for the generation of basic melodic shapes of the newborn cry. Results point out strengths and weaknesses of each method, thus suggesting their most appropriate use according to the goals of the analysis.

Two more papers propose methods to compute $\mathrm{F} 0$ and the main irregularity indexes of the voice (jitter, shimmer and HNR) for two different biomedical applications.

The paper from Andrea Guidi et al. "Features of vocal frequency contour and speech rhythm in bipolar disorder" concerns patients that undergo mood swings ranging from mania to depression. The goal is to develop a decision support system facilitating diagnosis and possibly predicting mood changes. A spectral analysis of F0contours extracted from audio recordings of a text read by bipolar patients and healthy control subject is reported. The algorithm is automatic and the obtained features describe speech rhythm and intonation. Feature trends are detected in bipolar patients across different mood states, while no significant differences are observed in healthy subjects.

The paper from Abdellah Kacha, "On the harmonic-to-noise ratio as an acoustic cue of vocal timbre of Parkinson speakers" addresses the relevance of the harmonic-to-noise ratio (HNR) and glottal cycle length jitter as cues of the vocal timbre of Parkinsonian speakers. Empirical mode decomposition is used to estimate the HNR by decomposing the log-magnitude spectrum of the speech signal into its harmonic, contour and noise components. Cycle length jitter has been estimated via the break-up by empirical mode decomposition of the cycle length time series into the intonation contour as well as the perturbations owing to tremor and jitter. HNR and cycle length jitter values of sustained vowels are evaluated in a large group of Parkinsonian and control speakers. Results show that the differences are not statistically significant between control and Parkinsonian speakers.

The following four papers make use of different devices and signals: EEG, acoustical signals and high-speed images.

The paper from Jaromir Horacek et al. "Low frequency mechanical resonance of the vocal tract in vocal exercises that apply tubes" concerns building a mathematical model of acoustic-structural interaction to clarify low frequency mechanical resonance of the vocal tract, that could enhance the effect of tube therapy in the context of phonation into a tube with the distal end in air and in water. The effects of phonation through the tube are demonstrated by registering oral air pressure and electroglottography, and by synchronous high speed filming of the water bubbling. The results show that the mechanical resonance can be near the measured water bubbling frequency $\mathrm{Fb}=11-11.5 \mathrm{~Hz}$.

The next paper from Marek Fric et al. "The effect of resonance tubes on facial and laryngeal vibration - a case study " exploits the effects of resonant tubes of different lengths and diameters measured by means of accelerometers placed on the subject's larynx, forehead and cheek. The electroglottographic (EGG) and acoustical signals were also recorded. The aim of the study was to find the frequency at which the resonance tubes have maximal effect and to find the experimental method for the estimation of the resonance frequencies of the elongated vocal tract. Results show two important maxima which can be identified as the maximum efficiency of the extended vocal tract (the maximum laryngeal vibration) and the correlation coefficient maximum between laryngeal vibration and the EGG signal.

The paper from Alberto Macerata et al., "Evaluation of the electroglottographic signal variability by amplitude-speed combined analysis", introduces a method for analyzing electroglottographic signals and for extracting features able to characterize phonation quantitatively. It is based on the EGG signal and its first derivative, which is related to the velocity of movements and contact of the vocal folds. For each glottal cycle the amplitude and related velocity signals are plotted in an $\mathrm{X}-\mathrm{Y}$ graph thus forming a multilayer display. The phonation process can thus be characterized in more detail by computing couples of indices (mean and variance) as obtained by dividing the graph in 4 quadrants, roughly associated with the different phases of the glottal cycle. The method proved to be efficient to discriminate normal subjects from pathological ones.

Finally the paper from Philipp Aichinger et al. "Fundamental frequency tracking in diplophonic voices" proposes two independent approaches for F0 tracking in diplophonic voices which are a special class of disordered voices characterized by multiple oscillators with different F0 active simultaneously. High-speed videos were obtained in addition to audio recordings. Promising qualitative and quantitative agreement of audio waveform modelling-based estimates with kymogram-based tracks was observed. The results illustrate that fundamental frequencies of diplophonic voices may be validly obtained from kymogram cycle marks, as well as via audio waveform modelling. The acquisition of two simultaneous F0 tracks in diplophonic voices might thus increase the validity of clinical voice analysis procedures.

The paper from Erick Fernando Gonzalez-Castañeda et al., "Sonification and textification: proposing methods for classifying unspoken words from EEG signals" exploits the use of EEG signals as an aid for patients affected by motor disorders. It makes use of EEG-based Brain-Computer Interfaces (BCI) within the emerging techniques of sonification and textification applied to EEG signals, which allows to characterize EEG signals as either an audio signal or a text document. The aim is to assess which of the two methods performs better in order to improve classification results of unspoken words. This might help to integrate people with severe motor disabilities to their environment. It is shown that the methods of sonification and textification of EEG improve the classification rates obtained using the EEG signals in their original form.

The last paper of this SI is from Philippe Dejonckere and Jean Lebacq: "Damping of vocal fold oscillation at voice offset". It exploits the damped oscillatory movement of the vocal folds while abducting at the end of a vocal emission. It reflects important mechanical properties of the vocal oscillator and cannot be voluntarily controlled, thus it could become a valuable clinical parameter, particularly in a medico-legal context. However its large variability in the same subject limits its use. Possibilities and limitations of high speed videoendoscopy and other glottographic methods are reviewed. Three main physiological factors accounting for the variability are analysed: the timing dynamics of the expiratory pressure with respect to the opening of the glottis; the speed at which vocal fold edges are abducted and glottal resistance drops; and the morphological change of the oscillator, whose lip-like shape becomes flattened depending on the degree of abduction. Additional research is required both as to the recording protocol and the development of dedicated software.

Reading these ten papers the interested reader will appreciate that, despite the quite different objectives, they have in common the rigorous approach, both theoretical and experimental, which is the basis for valuable scientific research. It is also worth underlying the use of different devices for signal acquisition, the multimodal approach and the development of innovative methods for the analysis of the recorded signals.

It is also worth pointing out the internationality of the authors that goes beyond Europe, as well as the interdisciplinary cooperation and the vastness of faced topics and issues, in line with the 
aims of the MAVEBA Workshop that has always stimulated and rewarded all this.

I am confident that this tradition will continue this year too at MAVEBA 2017 (http://maveba.dinfo.unifi.it/) and that it will still grow in the future.

Last but not least, I wish to express a special thanks to the anonymous reviewer that have freely devoted their time and competence to the review of the papers contributing to the success of this SI.
Claudia Manfredi

Biomedical Engineering Lab., Department of Information Engineering, Università degli Studi di Firenze, Firenze, Italy 